\title{
Community-acquired Pneumonia with Methicillin-resistant Staphylococcus Aureus in a Patient Admitted to the Intensive Care Unit: A Therapeutic Challenge
}

John Pham ${ }^{1}$, Talal Asif ${ }^{2}$, Majdi S. Hamarshi ${ }^{3}$

1. Department of Internal Medicine, University of Missouri Kansas City (UMKC) 2. Cardiology, John H. Stroger Hospital of Cook County, Chicago, USA 3. Department of Critical Care Medicine, Saint Lukes Hospital, University of Missouri Kansas City (UMKC)

$\square$ Corresponding author: Talal Asif, asift@umkc.edu

Disclosures can be found in Additional Information at the end of the article

\section{Abstract}

Methicillin-resistant Staphylococcus aureus (MRSA) was previously considered a purely nosocomial pathogen. However, community-acquired MRSA has recently emerged as an important cause of severe necrotizing community-acquired pneumonia (CA-MRSA) in previously healthy individuals. This new pathogen exhibits antibiotic resistance and is linked to extended hospital stay and higher mortality. CA-MRSA has presented new therapeutic challenges due to high vancomycin treatment failure and lack of specificity of clinical findings. There is emerging evidence that treatment with linezolid leads to better patient outcomes in patients with CA-MRSA. Through this case, we aim to raise awareness about early institution of therapy for CA-MRSA whenever it is suspected, to improve patient outcomes.

Categories: Internal Medicine, Infectious Disease, Pulmonology

Keywords: community acquired mrsa, linezolid, mrsa

\section{Introduction}

Linezolid and vancomycin are both recommended for treatment of methicillin-resistant Staphylococcus aureus (MRSA) pneumonia by most clinical societies. Some prospective randomized trials showed that the clinical and microbiological response of hospital or ventilator-associated pneumonia might be more favorable with linezolid as compared to vancomycin [1]. Case reports, experts, and at least one randomized clinical trial also suggest using linezolid in community-acquired pneumonia secondary to MRSA [1, 2]. Linezolid's possible superiority over vancomycin stems from the fact that it has better penetration into lung parenchyma, better availability at the tissue level, predictable blood level with kidney disease, and inhibition of toxin production in toxin-producing strains as in communityacquired MRSA (CA-MRSA) [1]. This case report describes the clinical course of a patient with community-acquired pneumonia secondary to toxin-producing CA-MRSA.

\section{Case Presentation}

A 41-year-old female patient with a past medical history of bipolar disorder, cocaine use disorder, and tobacco use disorder, presented with increased respiratory distress and combativeness. On initial examination, her vital signs included a temperature of $100.9^{\circ} \mathrm{F}$, heart rate of 140 beats per minute, respiratory rate of 30 breaths per minute and blood pressure of

\section{How to cite this article}

Pham J, Asif T, Hamarshi M S (January 03, 2018) Community-acquired Pneumonia with Methicillinresistant Staphylococcus Aureus in a Patient Admitted to the Intensive Care Unit: A Therapeutic Challenge. Cureus 10(1): e2019. DOI 10.7759/cureus.2019 


\section{Cureus}

103/66 mmHg. On physical examination, she was only able to speak in two-word sentences. There were bilateral coarse crackles all over the chest. She did not have any focal neurological deficits. Cardiovascular examination did not reveal abnormal heart sounds. Abdominal examination was unremarkable. The patient was immediately placed on a nonrebreather mask. However, the patient kept taking off her nonrebreather mask, continued to have worsening hypoxia, and eventually required tracheal intubation. Chest radiograph showed right upper lobe pneumonia (Figure 1).

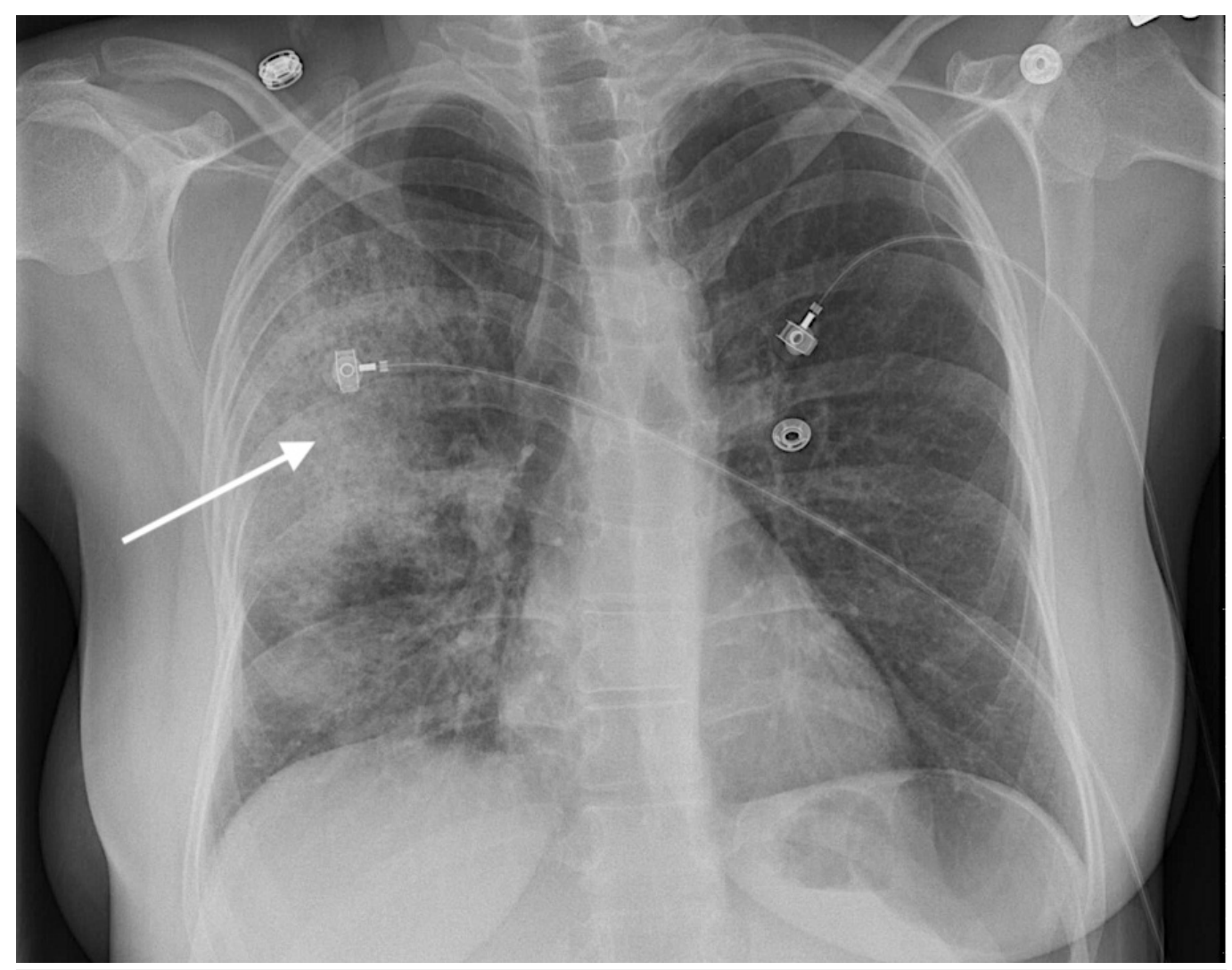

FIGURE 1: Patient's initial chest X-ray with arrow depicting right upper lobe pneumonia.

She was started on vancomycin, piperacillin-tazobactam and levofloxacin, and was transferred to the intensive care unit (ICU). Broad spectrum antibiotic therapy was chosen due to patient's critical condition and unclear etiology of the infecting pathogen. The patient had a white cell count of $11.8 \times 10^{3} /$ microliter $(\mu \mathrm{L})$ that trended to $2.2 \times 10^{3} / \mu \mathrm{L}$, normal troponins, and a lactic acid of $1.8 \mathrm{mmol} / \mathrm{Liter}(\mathrm{L}$ ) initially that trended up to $2.5 \mathrm{mmol} / \mathrm{L}$. Urine drug screen was positive for cocaine, benzodiazepine, and opiates. Urine Legionella antigen and Streptococcus pneumoniae antigen were negative. Influenza A was positive on rapid antigen testing and the patient was started on oseltamivir. This raised our concern for secondary bacterial pneumonia from MRSA as a complication of influenza virus infection. The patient had computed tomography (CT) of the chest that showed right upper, middle, and lower lobe consolidations with cystic destruction of the lung parenchyma (Figures 2, 3). 


\section{Cureus}

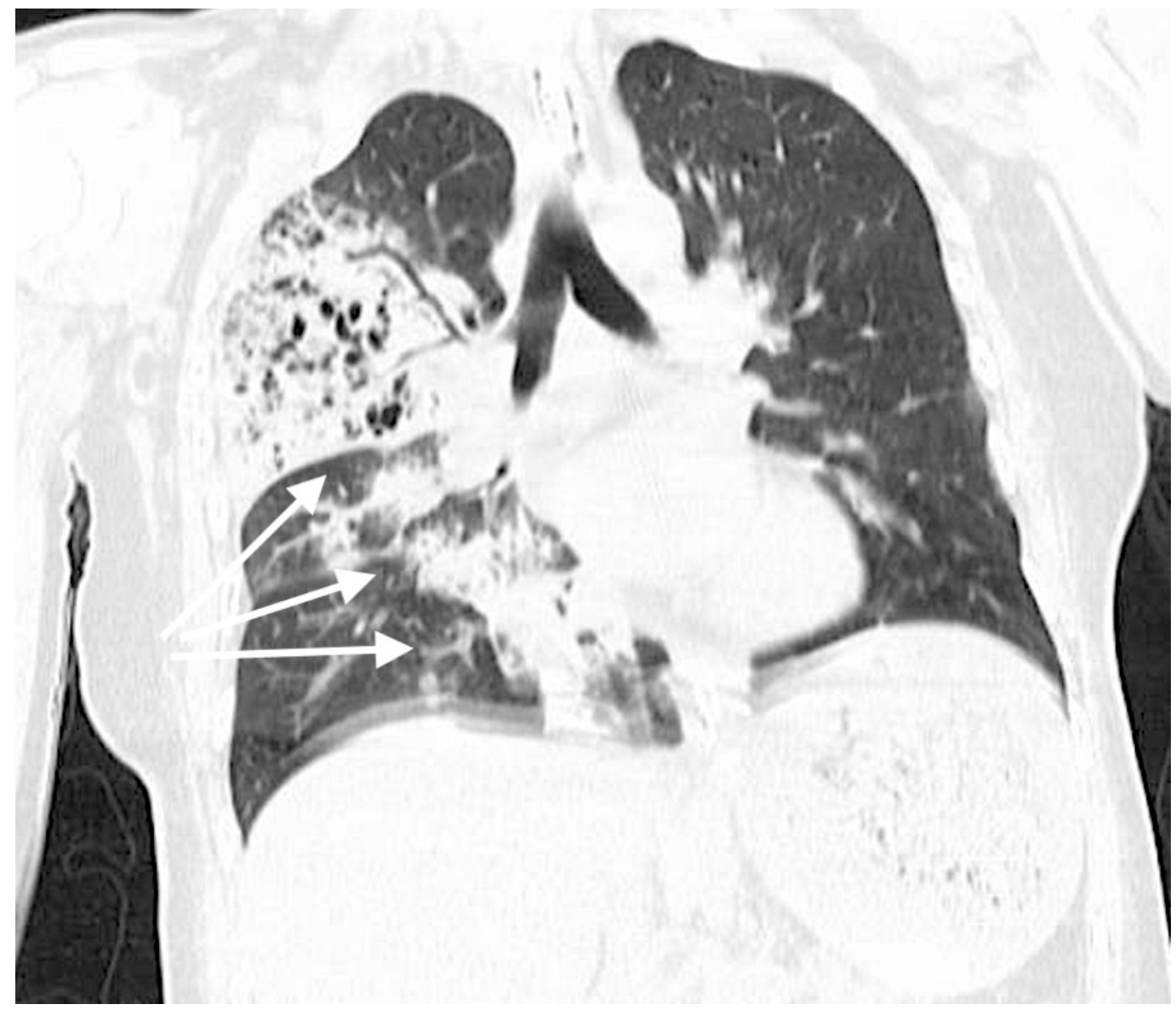

FIGURE 2: Computed tomography (CT) chest (coronal view) showing right upper, middle, and lower lobe consolidations (arrows).

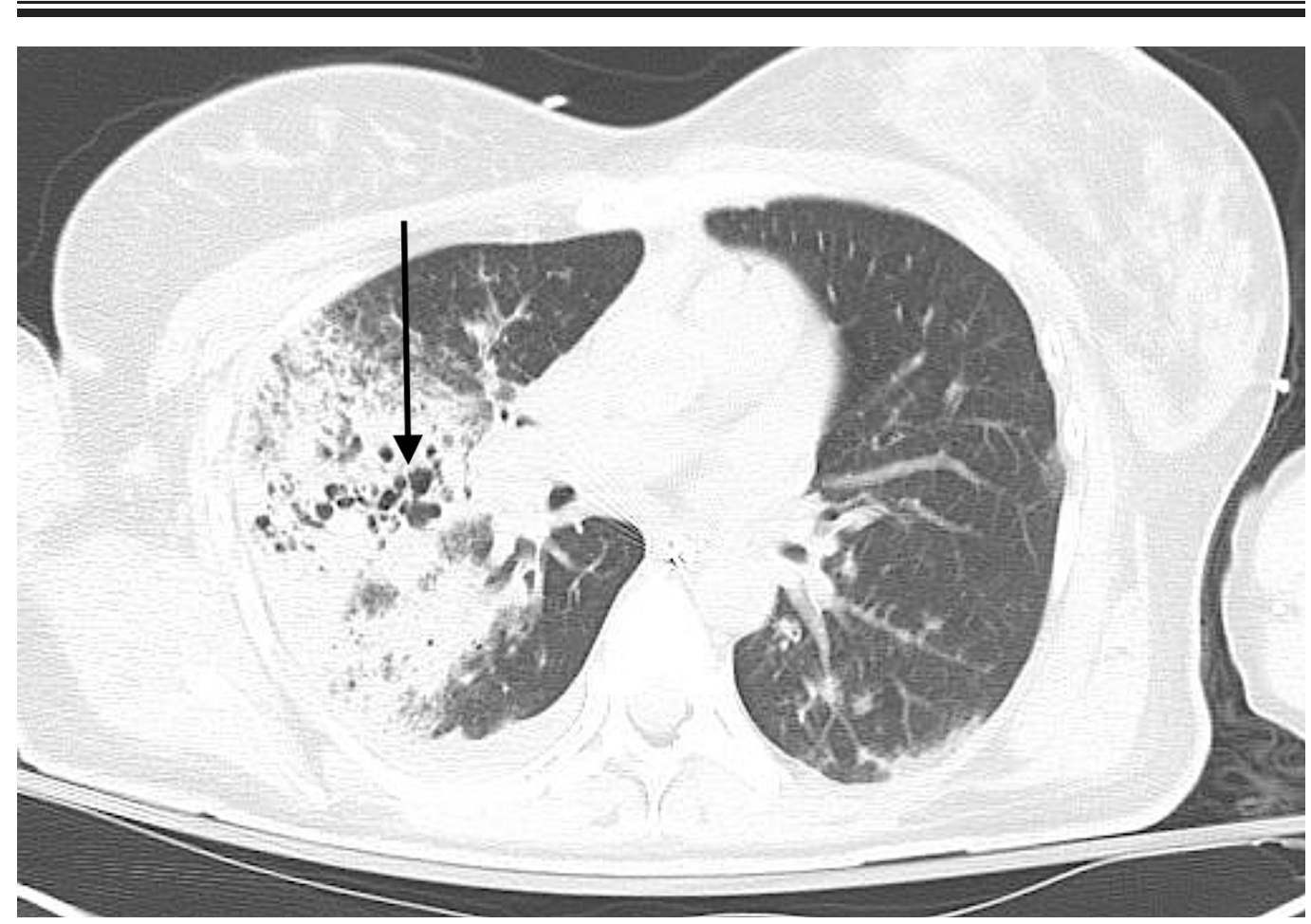




\section{Cureus}

\section{FIGURE 3: Computed tomography (CT) chest (axial view) showing cystic destruction of the lung parenchyma (arrow).}

Sputum culture and blood cultures grew MRSA that was sensitive to both vancomycin and clindamycin, confirming our diagnosis. Vancomycin had already been started on admission and was continued. Fluid resuscitation was also done early in the course, but eventually progressed to septic shock. The patient was then started on norepinephrine infusion and vasopressin infusion for blood pressure support. She then developed acute respiratory distress syndrome (ARDS). Her hypoxia became refractory to conventional ventilation, and extracorporeal membrane oxygenation (ECMO) was considered. She had a transesophageal echocardiogram which showed severe biventricular systolic dysfunction, global hypokinesis with an ejection fraction (EF) of 5\%. Her cardiomyopathy was felt to be acute and related to sepsis. She was managed for septic and cardiogenic shock, and venous-arterial ECMO was started. She was also started on continuous renal replacement therapy (CRRT) for hypervolemia refractory to diuresis, inotropic and vasopressor support. A follow-up transthoracic echocardiogram (TTE) five days later showed that the EF recovered to $45 \%$. She continued to remain intubated for 16 days and a repeat CT chest showed worsening and multiple cavitary lesions in the right lung (Figures 4, 5).

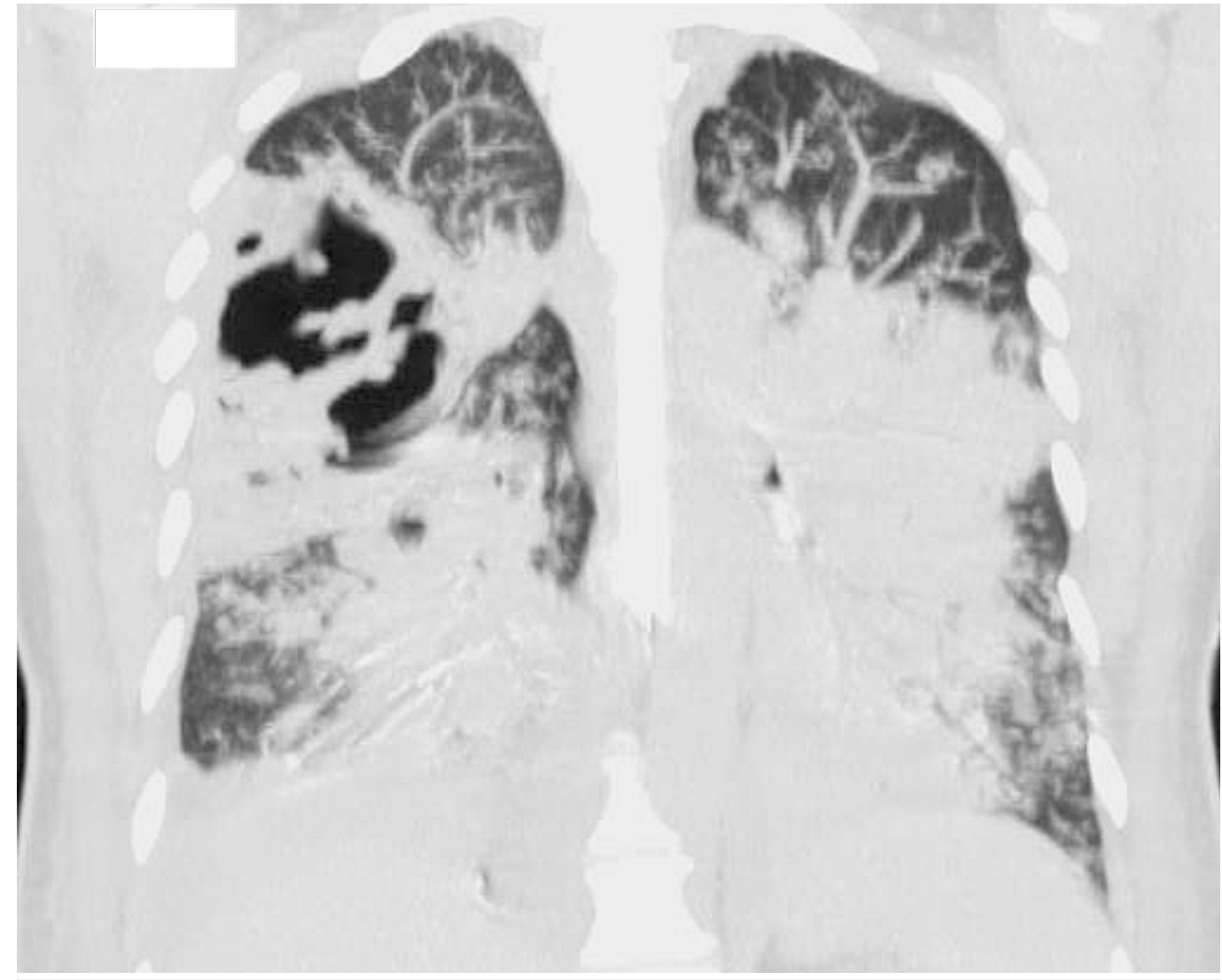

FIGURE 4: Repeat computed tomography (CT) chest (coronal view) showing worsening bilateral pneumonia. 


\section{Cureus}

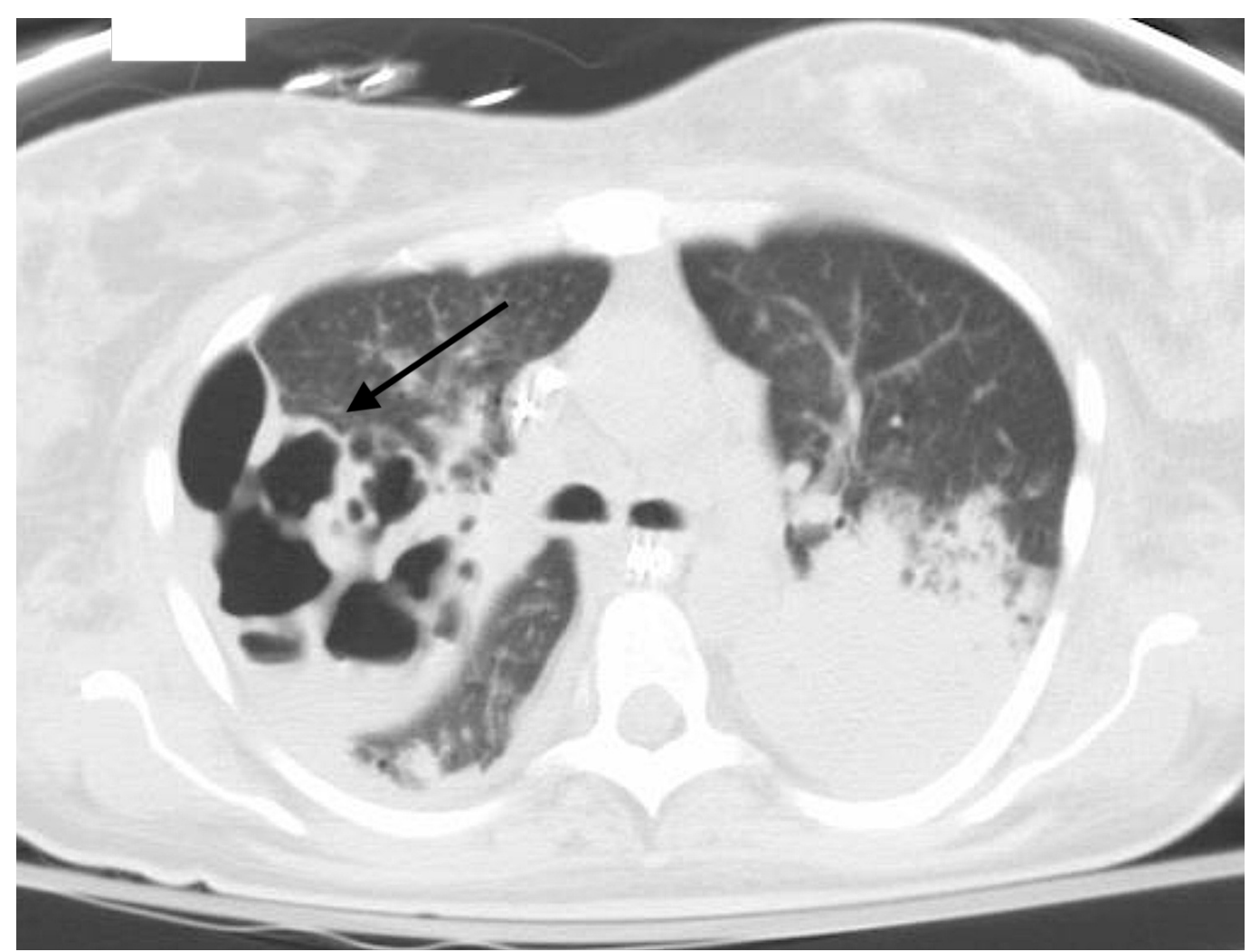

FIGURE 5: Computed tomography (CT) chest (axial view) showing worsening and multiple cavitary lesions in the right lung (arrow).

A repeat TTE showed EF of $60 \%$ and normal left and right heart function. ECMO was stopped and she was decannulated on day 9, CRRT was stopped on day 9. Unfortunately, she did not tolerate withdrawal of ECMO and continued to deteriorate and became difficult to wean from the ventilator. Given her lack of response to vancomycin, she was switched to daptomycin and linezolid, and the vancomycin was discontinued. She did not improve with new antibiotic regimen, continued to accumulate volume, and could not tolerate being off CRRT that was resumed for volume removal. Her hypoxia continued to be refractory despite all efforts. The family eventually decided to transition to comfort care due to her poor prognosis.

\section{Discussion}

MRSA can cause community-acquired pneumonia, and needs to be considered in the at-risk group of patients. This includes patients with known colonization with MRSA or those who have risk factors for colonization with MRSA (e.g., end-stage renal disease, contact sport participants, injection drug users, those living in crowded conditions, men who have sex with men, prisoners), recent influenza virus infection and antimicrobial therapy particularly with a fluoroquinolone in the prior three months. Also, it needs to be considered in communityacquired pneumonia presenting with necrotizing or cavitary lesions as in our patient, or with empyema. MRSA coverage should also be considered in community-acquired pneumonia severe enough to cause septic shock or respiratory failure requiring mechanical ventilation.

The most common CA-MRSA strain is USA300 [3]. Its pathogenicity appears to be greatly influenced by the Panton-Valentin-Leukocidin (PVL) toxin that causes leukocyte destruction, and also appears to play a major role in necrotizing pneumonia [3, 4]. The 2016 Infectious 
Disease Society of America recommendations regarding community-acquired pneumonia caused by MRSA or healthcare-associated pneumonia caused by MRSA is to treat with intravenous (IV) vancomycin or linezolid 600 milligrams (mg) twice daily either IV or oral [5]. While vancomycin has traditionally been used empirically for MRSA, it might have inadequate concentration in the lung tissue [6], tends to be under dosed in chronic kidney disease, might cause nephrotoxicity especially when combined with piperacillin-tazobactam, and it does not inhibit toxin production which is an important virulent factor in CA-MRSA pneumonia. On the other hand, linezolid appears to have greater lung penetration than vancomycin [3], does not need dose adjustment in patients with kidney disease, has great oral bioavailability and can be switched to oral regimen, and inhibits toxin production. Linezolid is the first of the oxazolidinone antibiotic that works by binding to the $50 \mathrm{~S}$ ribosomal subunit, resulting in the inhibition of protein synthesis. Linezolid appears to have activity against not just the PVL toxin, but against several additional toxins including alpha-hemolysin and alpha-type phenol soluble modulins, which contribute to its virulence [3]. Caution should be exercised with using linezolid given its association with myelosuppression, neurotoxicity, and serotonin syndrome, especially with patients on selective serotonin reuptake inhibitors, as well as lactic acidosis with high dose or prolonged therapy [7]. Some prospective randomized trials showed that linezolid is superior to vancomycin in regards to clinical and microbiological response in both nosocomial and community MRSA pneumonia [1]. One of the concerns regarding the use of linezolid would be the selective pressure towards linezolid-resistant Staphylococcus aureus. Therefore, it is important for clinicians to identify risk factors for MRSA as well as the patient's presentation. It is important to narrow the spectrum of antibiotic once susceptibility has been obtained, but it is just as important to monitor the patient's disease progression. Additional considerations include the use of molecular biology testing to allow rapid identification of MRSA in the sputum. One study has shown that the test helped to reduce the use and cost of anti-MRSA antibiotics in patients suspected of ventilator-associated pneumonia [8].

\section{Conclusions}

Accumulating body of evidence is suggesting that linezolid is superior to vancomycin in MRSA pneumonia in different clinical settings in terms of clinical response and microbiological clearance. However, there was no mortality benefit. Even though our patient was eventually switched to linezolid, unfortunately it was very late in the course. This case demonstrates the virulence of toxin-producing CA-MRSA, and consequent morbidity and mortality associated with CA-MRSA pneumonia. Given its high morbidity and mortality, clinicians might need to consider all measures that might help and as early as possible. We suggest using linezolid rather than vancomycin for CA-MRSA pneumonia, or at least adding a toxin inhibiting antibiotics in addition to vancomycin in the right clinical settings. We also suggest consideration of the diagnosis of CA-MRSA in patients with secondary bacterial pneumonia after influenza virus infection.

\section{Additional Information \\ Disclosures}

Human subjects: Consent was obtained by all participants in this study. Conflicts of interest: In compliance with the ICMJE uniform disclosure form, all authors declare the following:

Payment/services info: All authors have declared that no financial support was received from any organization for the submitted work. Financial relationships: All authors have declared that they have no financial relationships at present or within the previous three years with any organizations that might have an interest in the submitted work. Other relationships: All authors have declared that there are no other relationships or activities that could appear to have influenced the submitted work. 


\section{References}

1. Wunderink RG, Niederman MS, Kollef MH, et al.: Linezolid in methicillin-resistant Staphylococcus aureus nosocomial pneumonia: a randomized, controlled study. Clin Infect Dis. 2012, 54:621-629. 10.1093/cid/cir895

2. Chen J, Luo Y, Zhang S, et al.: Community-acquired necrotizing pneumonia caused by methicillin-resistant Staphylococcus aureus producing Panton-Valentine leukocidin in a Chinese teenager: case report and literature review. Int J Infect Dis. 2014, 26:17-21. 10.1016/j.ijid.2014.02.025

3. Kwong JC, Chua K, Charles PG: Managing severe community-acquired pneumonia due to community methicillin-resistant Staphylococcus aureus (MRSA). Curr Infect Dis Rep. 2012, 14:330-338. 10.1007/s11908-012-0254-8

4. Le VT, Le HN, Pinheiro MG, et al.: Effects of Tedizolid phosphate on survival outcomes and suppression of production of Staphylococcal toxins in a rabbit model of methicillin-resistant Staphylococcus aureus necrotizing pneumonia. Antimicrob Agents Chemother. 2017, 61:e02734-16. 10.1128/AAC.02734-16

5. Kalil AC, Metersky ML, Klompas M, et al.: Management of adults with hospital-acquired and ventilator-associated pneumonia: 2016 clinical practice guidelines by the Infectious Diseases Society of America and the American Thoracic Society. Clin Infect Dis. 2016, 63:e61-e111. 10.1093/cid/ciw353

6. Harigaya Y, Bulitta JB, Forrest A, et al.: Pharmacodynamics of vancomycin at simulated epithelial lining fluid concentrations against methicillin-resistant Staphylococcus aureus (MRSA): implications for dosing in MRSA pneumonia. Antimicrob Agents Chemother. 2009, 53:3894-3901. 10.1128/AAC.01585-08

7. Bishop E, Melvani S, Howden BP, et al.: Good clinical outcomes but high rates of adverse reactions during linezolid therapy for serious infections: a proposed protocol for monitoring therapy in complex patients. Antimicrob Agents Chemother. 2006, 50:1599-1602.

10.1128/AAC.50.4.1599-1602.2006

8. Dureau AF, Duclos G, Antonini F, et al.: Rapid diagnostic test and use of antibiotic against methicillin-resistant Staphylococcus aureus in adult intensive care unit. Eur J Clin Microbiol Infect Dis. 2017, 36:267-272. 10.1007/s10096-016-2795-5 